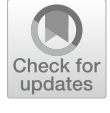

Cite as

Nano-Micro Lett.

(2022) $14: 14$

Received: 27 July 2021

Accepted: 8 October 2021

Published online: 6 December 2021

(C) The Author(s) 2021

\section{Air-Stable Ultrabright Inverted Organic Light-Emitting Devices with Metal Ion-Chelated Polymer Injection Layer}

\author{
Shihao Liu ${ }^{1,2}$, Chunxiu Zang ${ }^{1}$, Jiaming Zhang ${ }^{1}$, Shuang $\operatorname{Tian}^{2}$, Yan $\mathrm{Wu}^{2}$, Dong Shen ${ }^{2}$, \\ Letian Zhang ${ }^{1}$, Wenfa Xie ${ }^{1}$, Chun-Sing Lee ${ }^{2 凶}$
}

\title{
HIGHLIGHTS
}

- By using a zinc ion-chelated polyethylenimine as electron injection layer, an inverted organic light-emitting device (OLED) was successfully prepared with an external quantum efficiency over $10 \%$ at a brightness of $45,610 \mathrm{~cd} \mathrm{~m}^{-2}$.

- The chelation between zinc ion and amine groups not only passivates polar amine groups, but also fuses separated electronic orbitals.

- The inverted OLED show an excellent air stability with an operating time of $541 \mathrm{~h} @ 1,000 \mathrm{~cd} \mathrm{~m}^{-2}$ without any protection.

ABSTRACT Here, this work presents an air-stable ultrabright inverted organic lightemitting device (OLED) by using zinc ionchelated polyethylenimine (PEI) as electron injection layer. The zinc chelation is demonstrated to increase the conductivity of the PEI by three orders of magnitude and passivate the polar amine groups. With these physicochemical properties, the inverted OLED shows a record-high external quantum efficiency of $10.0 \%$ at a high brightness of $45,610 \mathrm{~cd} \mathrm{~m}^{-2}$ and can deliver a maximum brightness of $121,865 \mathrm{~cd} \mathrm{~m}^{-2}$. Besides, the inverted OLED is also demonstrated to possess an excellent air stability (humidity, 35\%) with a half-brightness operating time of $541 \mathrm{~h} @ 1000 \mathrm{~cd} \mathrm{~m}^{-2}$ without any protection nor encapsulation.

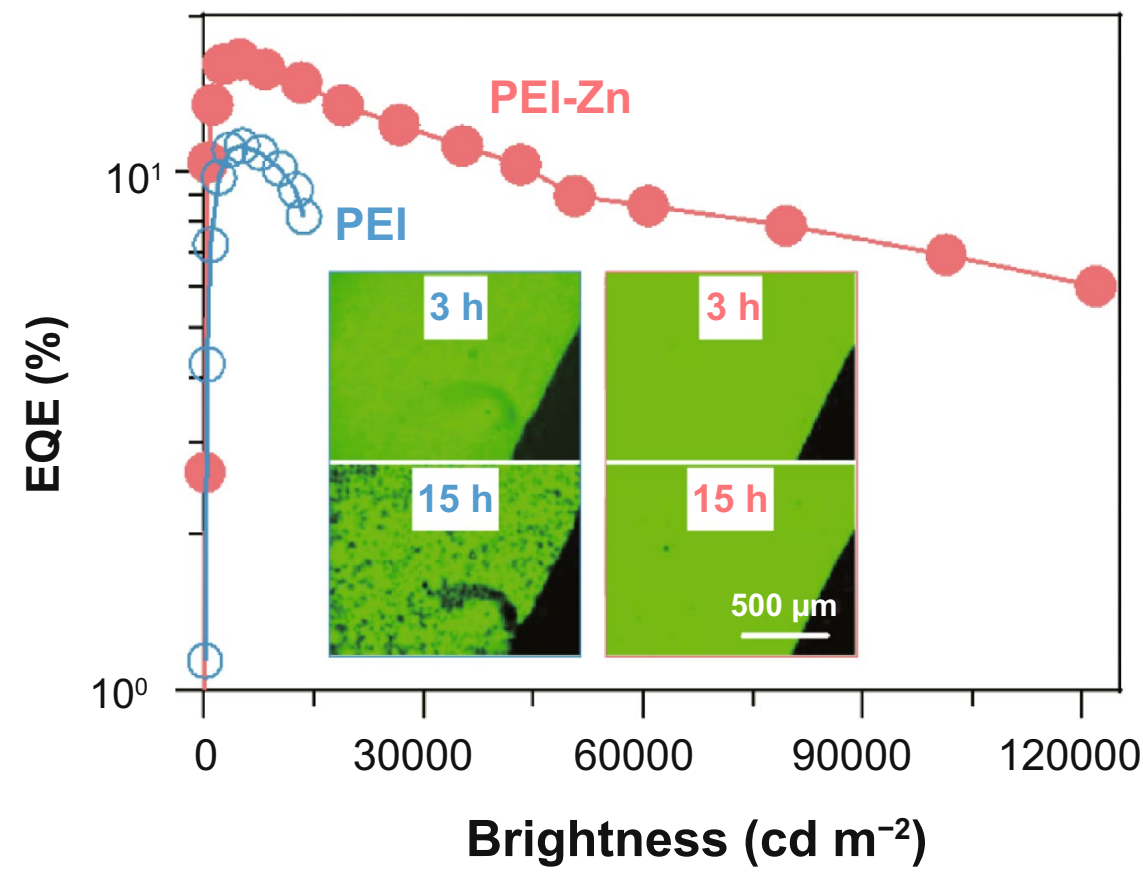

KEYWORDS Air stability; Ultrabright; Electron injection; Metal ion chelation; Inverted organic light-emitting device

Shihao Liu and Chunxiu Zang have contributed equally.

$\bowtie$ Wenfa Xie, xiewf@jlu.edu.cn; Chun-Sing Lee, apcslee@ cityu.edu.hk

1 State Key Laboratory of Integrated Optoelectronics, College of Electronics Science and Engineering, Jilin University, 2699 Qianjin Street, Changchun 130012, People's Republic of China

2 Department of Chemistry, Center of Super-Diamond and Advanced Films (COSDAF), City University of Hong Kong, Kowloon, Hong Kong, People's Republic of China 


\section{Introduction}

Organic light-emitting device (OLED) displays have gained momentum as displays for TV and smartphone in the past ten years [1]. With time goes on, information displays are no longer limited to flat panels that are just placed in front of the users but aimed at revolutionizing the way of interactions between the users and their surrounding environment [2-4]. Many new potential applications would demand high performance at high brightness. In particular, a brightness over 10,000 $\mathrm{cd} \mathrm{m}^{-2}$ is needed for see-through display (such as Google glass), augmented reality (AR) display and highdynamic range (HDR) display [2]. So far, most efforts have been made to achieve the theoretical maximum efficiency of OLEDs [5-8], whereas high-brightness performance is not getting as much attention. In order for OLEDs to be competitive with other light-emitting technologies, such as $\mu \mathrm{LED}$, high-brightness performance of OLEDs would have to be improved.

Moreover, reactive materials, such as alkali metals and salts, can be not used in inverted OLEDs with a bottom cathode. Inverted OLEDs are thus considered as an ideal structure for constructing air-stable OLEDs [9-13]. For example, it has been demonstrated that with a same encapsulation, a flexible display using inverted OLEDs can work after over 1 year, while that using conventional OLEDs cannot work normally after 21 days [13]. Due to high injection barrier (over $1 \mathrm{eV}$ ) from the metal oxides cathode to the organic layer, an electron injection interlayer (EJL) comprising amine-based molecules is used to promote electron injection in inverted OLEDs [12, 14-16]. In fact, inverted OLEDs with polyethylenimine (PEI) interlayer have achieved driving voltages and efficiencies comparable to the conventional devices [15]. However, highbrightness performances of OLEDs are limited by conductive properties of the amine-based polymers. It has to be noted that while most typical organic semiconductors have energy gap $\left(E_{\mathrm{g}}\right)$ around $3 \mathrm{eV}$, PEI is an insulator with an $E_{\mathrm{g}}$ over $5 \mathrm{eV}$ [16]. At high voltages, the PEI will become the main hurdle for improving high-brightness performance. As a result, ultrabright inverted OLEDs (over 100,000 $\mathrm{cd} \mathrm{m}^{-2}$ ) have rarely been reported so far.

Here, we report an air-stable ultrabright inverted OLEDs by using zinc ion-chelated PEI (PEI-Zn) as EJL. The chelation between zinc ion and amine group can fuse separated orbitals of two neighboring PEI chains into a molecular orbital. The conductivity of the Zn-chelated PEI is nearly three orders of magnitude higher than that of PEI. Besides, the chelation of zinc ions also reduces the chemical reactivity of the amine groups and thus suppresses the degeneration effect of PEI. Finally, an ultrabright inverted OLED with a record-high maximum brightness of $121,865 \mathrm{~cd} / \mathrm{m}^{2}$ is prepared by using PEI-Zn as EJL. The inverted OLED still shows an external quantum efficiency (EQE) over $10 \%$ at a high brightness of $45,000 \mathrm{~cd} \mathrm{~m}^{-2}$. Interestingly, without any protection nor encapsulation, the inverted OLED with PEI$\mathrm{Zn}$ also shows a half-brightness operating time of $541 \mathrm{~h} @$ $1000 \mathrm{~cd} \mathrm{~m}^{-2}$ in air (humidity, 35\%).

\section{Methods}

\subsection{Materials and Device Fabrication}

Polyethylenimine (PEI, Mw: 10,000) and zinc acetate dihydrate were purchased from Shanghai Aladdin Biochemical Technology Co. Ltd. $\mathrm{MoO}_{3}, 4,4^{\prime}-\mathrm{N}, \mathrm{N}^{\prime}$-dicarbazole (CBP), di-[4-(N,N-di(p-tolyl)-amino)-phenyl] cyclohexane (TAPC), 4,4',4"-Tris (carbazol-9-yl) triphenylamine (TCTA), 2,2',2"(1,3,5-benzinetriyl)-tris(1-phenyl-1-H-benzimidazole) (TPBi) and tris (2-phenylpyridine) iridium (III) (Ir(ppy) $)_{3}$ ) were obtained from Luminescence Technology Corporation. 10,10'-[5-(6-[1,1'-Biphenyl]-4-yl-2-phenyl-4-pyrimidinyl)1,3-phenylene] bis [9,10-dihydro-9,9-dimethyl-acridine] (DMAC-BPP) was synthesized by Beijing Tuocai Optoelectronics Technology Co. Ltd. All materials and solvents were used as received without further purification. To prepare PEI-Zn precursors, $0.0075 \mathrm{~g}$ zinc acetate dihydrate was added to the $1 \mathrm{~mL}$ PEI (0.1 wt\%, 2-methoxyethanol) solution and stirred until the solution became clear. A small amount $(40 \mu \mathrm{L})$ of water was added to facilitate dissolving the zinc acetate dihydrate. After that, the white PEI-Zn polymer was obtained by freeze-drying in reserve. Before devices fabrication, the ITO glass substrates were cleaned with Decon 90, ultrasonic cleaning in deionized water and dried in the oven. Then, the substrates were processed in a plasma cleaner chamber (PDC-32G, Harrick). The PEI solution or PEI-Zn precursor solution was spin-coated on substrates at $3000 \mathrm{rpm}$ for $40 \mathrm{~s}$, following a thermal annealing at $150{ }^{\circ} \mathrm{C}$ for $10 \mathrm{~min}$. Organic layers and anode were deposited sequentially by vacuum thermal evaporation process on the substrates under 
vacuum $\left(\sim 6 \times 10^{-4} \mathrm{~Pa}\right)$. The deposition rate of organic layers, $\mathrm{MoO}_{3}$ and $\mathrm{Ag}$ anode is $0.3-1,0.1-0.15$, and $1.5-2.5 \AA \mathrm{s}^{-1}$, respectively.

\subsection{Film and Device Characterizations}

Current density-voltage-luminance characteristics and EL spectra of unpackaged devices without any light outcoupling instruments were measured with a Goniophotometric Measurement System based on spectrometer (GP-500, Otsuka Electronics Co., Osaka, Japan) in the air at room temperature simultaneously. Ultraviolet and X-ray photoelectron spectrometer measurements were carried out with a highresolution photoemission spectrometer (R3000, PREVAC). Transient PL decay curves of the devices were measured using a IHR320 spectrometer (HORIBA, Japan). Transient EL measurements were carried out with an arbitrary waveform generator (Rigol, DG5102), and transient responses of the devices were obtained with a HOLITA fluorescence spectrum analyzer system (HOLITA Co. Beijing, China). Capacitance-voltage curves were obtained with an impedance analyzer (TH2829C, Tonghui Co., Changzhou, China). Operating lifetime of the unpackaged devices was measured by using an OLED aging tester (ZJZCL-1, Shanghai University, China) under a constant current in atmospheric conditions. Images of light-emitting areas of devices were obtained by fluorescence microscope (Mshot M53, Guangzhou, China). The FTIR spectra were measured by using a Nicolet iS50 FTIR Spectrometer. Electrochemical measurements were performed using an IVIUMnSTAT multichannel electrochemical analyzer. A three-electrode electrochemical cell consisted of a platinum-connected ITO electrode as the working electrode, an $\mathrm{Ag} / \mathrm{AgCl}$ (vs. $\mathrm{Ag} / \mathrm{AgCl}, 3 \mathrm{M} \mathrm{KCl}$ ) as the reference electrode, and a platinum plate as the counter electrode. $\mathrm{pH}$ values were measured by using a $\mathrm{pH}$ meter (PH808, Smart Sensor, Dongguan, China).

\section{Results and Discussion}

\subsection{Device Structure of Inverted OLED with PEI-Zn Electron Injection Layer}

Figure 1 shows device structures and energy level diagrams of inverted OLEDs with a PEI-Zn EJL or with a PEI EJL.
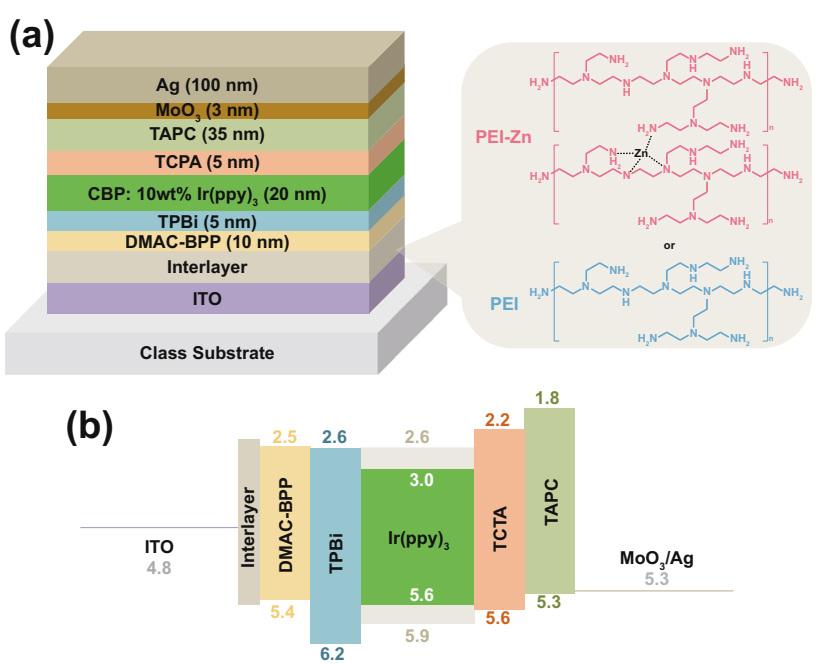

Fig. 1 a Device structure and b energy level diagram of an inverted OLED with a PEI-Zn or a PEI interlayer

Molecular structures of the used small molecular materials in the inverted OLEDs are shown in Fig. S1. Here, an inert Ag layer modified by $\mathrm{MoO}_{3}$ is used as the top refractive anode, while an ITO is used as the bottom transparent cathode. The emitting layer consists of a bipolar host CBP doped with a green phosphor $\left(\operatorname{Ir}(\mathrm{ppy})_{3}\right)$. The PEI-Zn or the PEI interlayer is deposited between the bottom ITO cathode and electron transport layer. With reference to the previous work, a 15-nm PEI or PEI-Zn interlayer is used in this work. It is because a thicker amine-containing polymer layer did not further reduce the working function and would adversely affect device performances due to its insulating natures [16]. The PEI-Zn in this work is synthesized by following the reported method [17], and the obtained sample is a white polymer (Fig. S2). Besides, effects of using different amount of zinc acetate dihydrate were also studied (Fig. S3). Compared to organic solar cells, active layers of OLEDs always have much deeper lowest unoccupied molecular orbital (LUMO) [18-20]. Thus, device performances of OLEDs are more sensitive to the working function of the interlayer. The $0.037 \mathrm{~g}$ of zinc acetate dihydrate in Zhou's work will lead to a working function of $4.1 \mathrm{eV}$ [17], and thus poor electron injection in OLEDs (see Fig. S3). The amount of zinc acetate dihydrate was thus reduced to $0.0037 \mathrm{~g}$ to ensure efficient electron injection and enhanced conductivity. With these parameters, efficient inverted OLEDs were prepared in this work. 


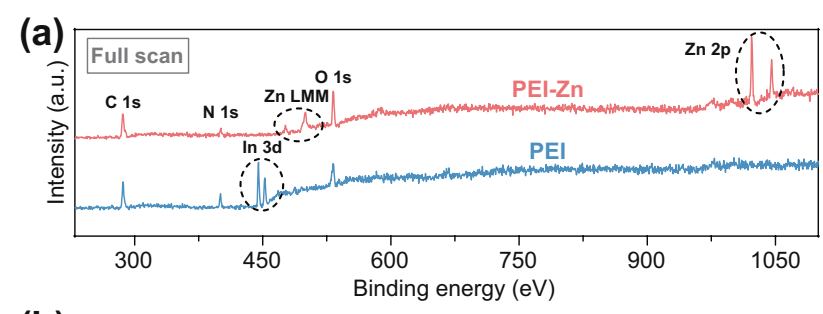

(b)
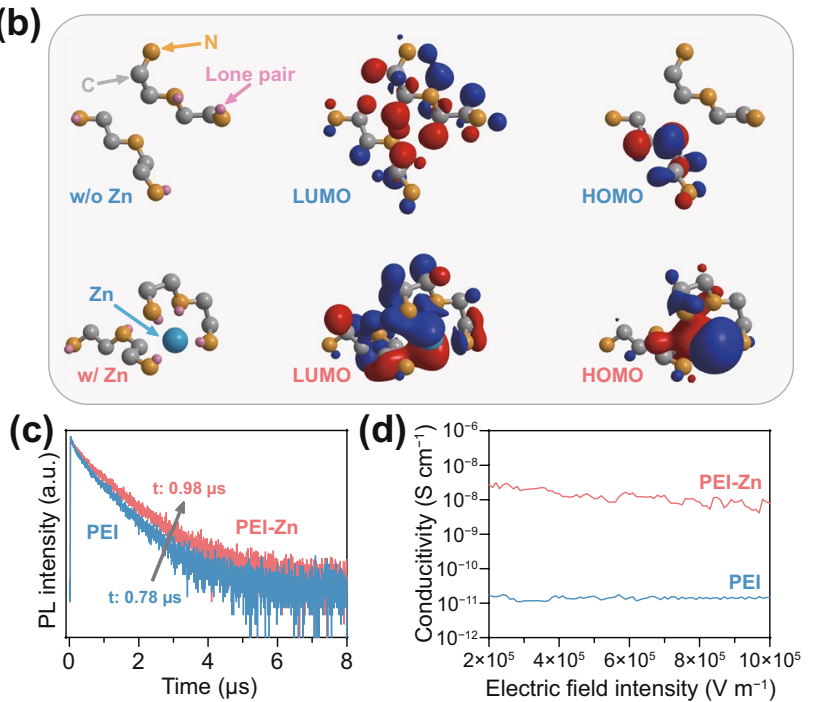

Fig. 2 a XPS spectra of a PEI and a PEI-Zn layers coated on ITO substrates. b Optimized structure and molecular orbitals of two enamine molecules with and without zinc ions. c Transient PL characteristics (@520 nm) of green emitting layer (CBP:10 wt\% $\operatorname{Ir}(\mathrm{ppy})_{3}$ ) deposited, respectively, on PEI and PEI-Zn. d Conductivity of PEI and PEI-Zn

\subsection{Characteristics of PEI-Zn Film}

Firstly, properties of the PEI-Zn coated on ITO are investigated as shown in Fig. 2 and S4-S9. Figure 2a shows X-ray photoelectron spectroscopy (XPS) results of a PEI-Zn and a PEI layer coated on ITO substrates. The Zn $2 p$ (1021 and $1045 \mathrm{eV}$ ) and $\mathrm{Zn}$ LMM (476 and $500 \mathrm{eV}$ ) peaks can be clearly seen in the PEI-Zn sample. The N1s peak in PEI$\mathrm{Zn}$ shifts to a higher binding energy (Fig. S4) compared to that of PEI. As shown in the Fourier transform infrared (FTIR) spectra (Fig. S5), the pristine PEI shows the stretching vibration of the $-\mathrm{NH}_{2}$ at $3360 \mathrm{~cm}^{-1}$ and the bending vibration of the $-\mathrm{NH}_{2}$ at $1664 \mathrm{~cm}^{-1}$, while the PEI-Zn does not. Besides, the $-\mathrm{NH}$ stretching vibration at $3285 \mathrm{~cm}^{-1}$ and bending vibration at $1600 \mathrm{~cm}^{-1}$ of the PEI are also found to shift to lower wavenumbers in the PEI-Zn. These results confirm the chelation between $\mathrm{Zn}$ ions and the polar amine groups (- $\mathrm{NH}_{2}$ and $\left.-\mathrm{NH}\right)$ of the PEI chains. Interestingly, we also observe the In $3 \mathrm{~d}$ (445 and $452 \mathrm{eV}$ ) peaks in PEI, but not in PEI-Zn (Fig. 2a). It is considered as a result of the passivated reactivity of the PEI-Zn compared to the PEI. As we also see from their $0.1 \mathrm{wt} \%$ aqueous solution, the $\mathrm{pH}$ (10.3) of the PEI solution is much higher than that (6.9) of the PEI-Zn solution. The absence of In $3 \mathrm{~d}$ peaks in PEI-Zn indicates that the chelation between amine groups and $\mathrm{Zn}$ ions can avoid the corrosion to the ITO during the preparation of amine-based layers. Besides, following the method in the previous work [21], we measured the electrochemical stability of the PEI and the PEI-Zn films. As shown in Fig. S6, the PEI-Zn film also shows an enhanced electrochemical stability compared to the PEI film. The operating lifetime of inverted OLEDs with PEI-Zn is thus considered to benefit from these properties of the PEI-Zn, and the evidence is shown below in this paper.

The chelation between $\mathrm{Zn}$ ions and amine groups can also efficiently enhance the conductivity and passivate the polar amine groups of PEI (Fig. 2b-d). We take molecular mechanics calculations using a model of chained enamine molecule to represent the PEI chain for simplicity. The optimized structures (left of Fig. 2b) and the molecular orbitals (middle and right of Fig. 2b) of two enamine molecules with and without zinc ions are obtained from the theoretical calculations. We can see that after adding a zinc ion, some electron lone pairs of amine groups are attracted by the zinc ions, corresponding to the increase in the binding energy of the $\mathrm{N} 1 \mathrm{~s}$ (Fig. S4). It is reasonably considered that the rearrangement of the polar amine groups can obviously reduce the polarity of the PEI-Zn as the rearranged dipoles could cancel each other. Interestingly, the chelation effect also enhances the electronic coupling between the two enamine molecules. As we seen from Fig. 2b, there are only separated molecular orbitals in the two enamine molecules without zinc ion, while the molecular orbitals are combined into a fused orbital after adding a zinc ion. According to these theoretical results, the polarity and resistivity of the PEI-Zn should be both reduced compared to those of PEI due to the rearrangement of the polar amine groups and the orbitals fusion. To experimentally verify these, we deposited green organic emitting films (CBP:10 wt\% $\operatorname{Ir}(\text { ppy })_{3}$ ) on a PEI and a PEI-Zn films and measured their transient EL characteristics (Fig. 2c). Figure 2c shows that the exciton lifetime of the green emitter increases from $0.76 \mu$ s on the PEI to $0.98 \mu$ s on the PEI-Zn. In fact, the pristine exciton lifetime of the $\operatorname{Ir}(\mathrm{ppy})_{3}$ should be about $1 \mu$ s [22]. This 
indicates that the PEI has a serious quenching effect due to serious dipole-dipole interactions, while the PEI-Zn has negligible. The conductivities of the PEI and PEI-Zn are also measured and shown in Fig. 2d. The conductivities are obtained by measuring the voltage-current characteristics of a 15-nm-thick PEI or PEI-Zn on an ITO grid (Fig. S7). Because of the low working functions of PEI and PEI-Zn (Fig. S8), it can be reasonably considered that only electrons inject from ITO and transport through the PEI or the PEI-Zn films, and the conductivities of Fig. $2 d$ belong to electron conductivities rather than the overall charge conductivities. As seen in Fig. 2d, the electron conductivity of the PEI-Zn is nearly three orders of magnitude higher than that of the PEI. Tauc plots (Fig. S9) also reveal a reduction of optical energy gap from $5.2 \mathrm{eV}$ of the PEI to $3.6 \mathrm{eV}$ of the PEI$\mathrm{Zn}$. The reduced energy gap of the PEI-Zn can lead to an increase in carrier density. Moreover, combined the fact that the structural building blocks of PEI chains are held together by comparatively weak van der Waals forces, the chelation can also enhance carrier mobility with the fused molecular orbital (Fig. 2b). Considering the above, the enhanced conductivity of the PEI-Zn is a result of the combination of its enhanced carrier density and mobility. Nevertheless, because the pristine PEI has a high cationic charge-density
[16], it is reasonable to consider the enhanced carrier mobility as the major reason of the enhanced conductivity of the PEI-Zn. It should also be noted that the passivation and the electronic coupling of the PEI chains are not achieved by sacrificing their electron injection ability. As we see from the photoemission cutoff obtained via UPS spectra (Fig. S8), the working function varies by less than $0.1 \mathrm{eV}$ before and after adding the zinc ions.

\subsection{Performances of OLED with PEI-Zn EJL}

Two inverted OLEDs (Fig. 1a, b) using PEI or PEI-Zn as EJL are, respectively, prepared and marked as device PEI and device PEI-Zn. Current density-voltage-brightness, current efficiency-brightness-power efficiency, and EQE-brightness characteristics of the two devices are shown in Fig. 3a-c. Figure 3 a shows that the two devices show similar current density-voltage characteristics below $8 \mathrm{~V}$, while they behave differently at higher voltages. Device PEI-Zn shows much higher current density at the voltage over $8 \mathrm{~V}$, and even works well after device PEI breaks down (@10 V). Due to their current density-voltage characteristics, device PEI shows a maximum brightness of about $15,000 \mathrm{~cd} \mathrm{~m}^{-2}$, which is at the same level with other reported inverted OLEDs (a)
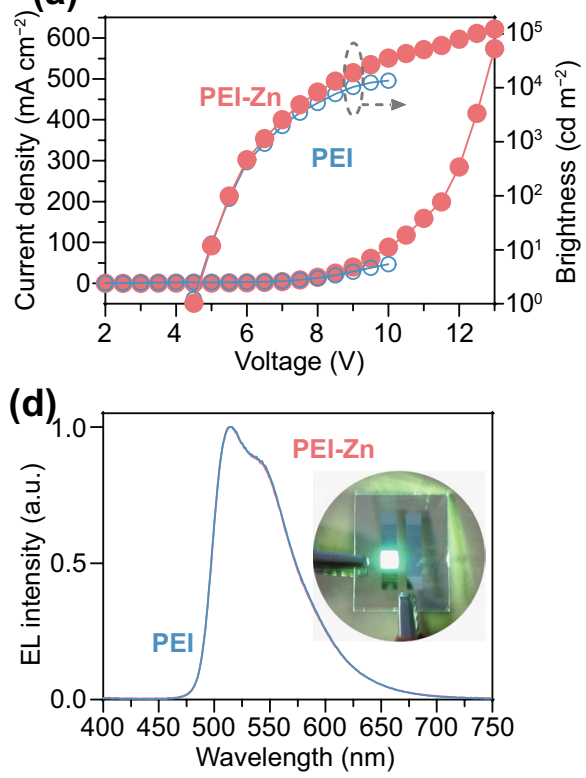

(b)

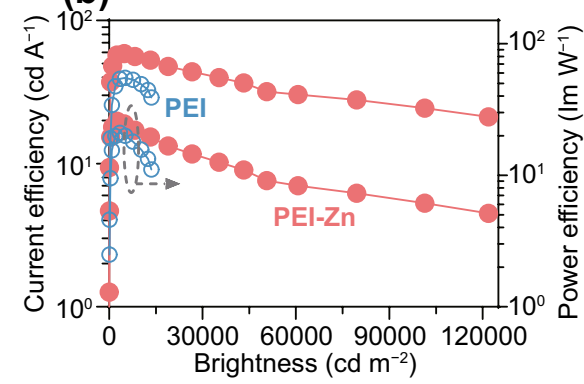

(e)

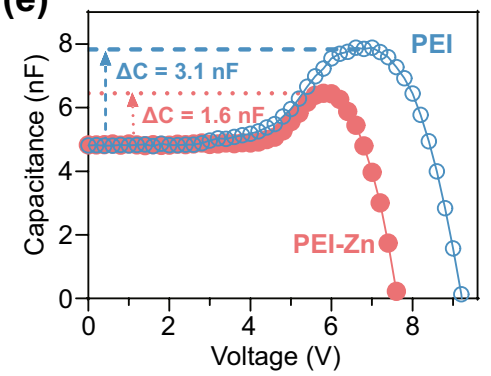

(c)

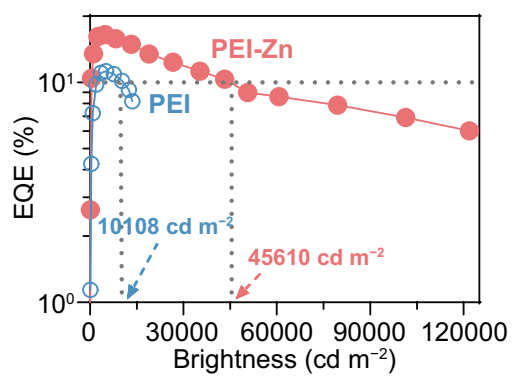

(f)

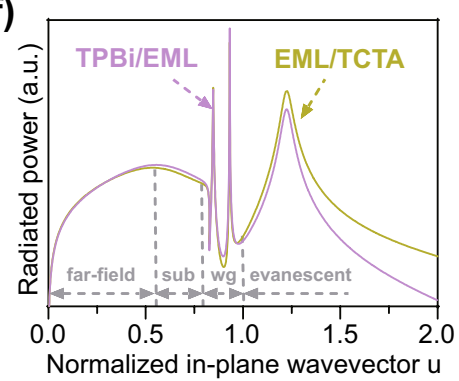

Fig. 3 a Current density-voltage-brightness, b current efficiency-brightness-power efficiency, c EQE-brightness, d EL spectra and e capacitance-voltage characteristics of device PEI and device PEI-Zn, $\mathbf{f}$ power dissipation spectra of dipole sources at the EML/TCTA interface and the TPBi/EML interface 
Table 1 Device performances of inverted OLEDs

\begin{tabular}{|c|c|c|c|c|c|c|c|c|}
\hline \multirow[t]{2}{*}{$\overline{\mathrm{ETL}}$} & \multirow{2}{*}{$\begin{array}{l}\mathrm{V}_{\mathrm{T}}^{\mathrm{a}} \\
(\mathrm{V})\end{array}$} & \multirow{2}{*}{$\begin{array}{l}\text { Maximum } \\
\text { Brightness } \\
\left(\mathrm{cd} / \mathrm{m}^{2}\right)\end{array}$} & \multicolumn{6}{|c|}{$@ 10,000 \mathrm{~cd} \mathrm{~m}^{-2}$} \\
\hline & & & $\begin{array}{l}\mathrm{CE}^{\mathrm{b}} \\
(\mathrm{cd} / \mathrm{A})\end{array}$ & $\begin{array}{l}\mathrm{PE}^{\mathrm{c}} \\
(\mathrm{lm} / \mathrm{W})\end{array}$ & $\begin{array}{l}\mathrm{EQE} \\
(\%)\end{array}$ & $\begin{array}{l}\mathrm{CE} \\
(\mathrm{cd} / \mathrm{A})\end{array}$ & $\begin{array}{l}\mathrm{PE} \\
(\mathrm{lm} / \mathrm{W})\end{array}$ & $\begin{array}{l}\text { EQE } \\
(\%)\end{array}$ \\
\hline PEI-Zn (this work) & 4.5 & 121,866 & 58.6 & 25.8 & 16.5 & 55.3 & 18.0 & 15.5 \\
\hline PEI (this work) & 4.5 & 13,566 & 39.8 & 16.4 & 11.2 & 33.3 & 12.6 & 10.5 \\
\hline $\mathrm{ZnO} / \mathrm{PEI}$ & 2.6 & 38,932 & 31.1 & 19.5 & 9.1 & 23.7 & 10.0 & 6.6 \\
\hline $\mathrm{ZnO} / \mathrm{Bphen}: \mathrm{CsCO}_{3}$ (Ref. [23]) & 3.4 & $\sim 4500$ & 52.5 & N/A & 14.3 & 33.4 & N/A & 9.3 \\
\hline ZnO/s-SPB + N-DMBI (Ref. [26]) & 3.0 & $\sim 4000$ & N/A & N/A & $\sim 15$ & N/A & N/A & N/A \\
\hline $\mathrm{ZnO} / \mathrm{PEI} / \mathrm{Cs}_{2} \mathrm{CO}_{3}: \mathrm{Alq}_{3}$ (Ref. [10]) & N/A & $\sim 35,000$ & 53.3 & 40.8 & 19.7 & $\sim 20.0$ & $\sim 10.0$ & $\sim 11.0$ \\
\hline $\begin{array}{l}\mathrm{Mg} \text { doped } \mathrm{ZnO} / \mathrm{PEIE}: \mathrm{Rb}_{2} \mathrm{CO}_{3} \text { (Ref. } \\
\text { [30]) }\end{array}$ & 3.6 & 31,540 & 65.5 & 43.0 & N/A & 57.7 & 27.8 & N/A \\
\hline
\end{tabular}

${ }^{\mathrm{a}}$ The voltage at the brightness of $1 \mathrm{~cd} \mathrm{~m}^{-2}$

${ }^{\mathrm{b}}$ Current efficiency

${ }^{\mathrm{c}}$ Power efficiency

$[9,10,12,13,23-30]$, but device PEI-Zn shows a recordhigh brightness of $121,865 \mathrm{~cd} \mathrm{~m}^{-2}$. Besides, as shown in Fig. 3b, c, device PEI-Zn also has higher efficiencies than device PEI across the whole brightness range. For example, at the brightness of $5000 \mathrm{~cd} \mathrm{~m}^{-2}$, the EQE of device PEI-Zn is $16.4 \%$, while that of device PEI is only $11.1 \%$. More interestingly, the maximum brightness with a EQE over $10 \%$ can attain to $45,610 \mathrm{~cd} \mathrm{~m}^{-2}$ for device PEI-Zn, which is nearly 4.5 times of that $\left(10,108 \mathrm{~cd} \mathrm{~m}^{-2}\right)$ for device PEI. For comparison, device $\mathrm{ZnO} / \mathrm{PEI}$ with $\mathrm{ZnO}$ (30 nm)/ PEI $(15 \mathrm{~nm})$ as electron transport layer is also prepared (Fig. S10). Device performances of the inverted OLEDs of this work and the recently reported devices are summarized in Table 1. Compared to device PEI and device PEI-Zn, device $\mathrm{ZnO} / \mathrm{PEI}$ shows a higher current density and thus a lower turn-on voltage (@ $2.7 \mathrm{~V}$ ). The enhanced carrier injection of device $\mathrm{ZnO} / \mathrm{PEI}$ is attributed to the reduced working function of $\mathrm{ZnO}$-coated ITO (4.5 eV) [31]. Besides, device $\mathrm{ZnO} /$ PEI shows a maximum brightness of about $38,932 \mathrm{~cd} \mathrm{~m}^{-2}$, which is two times higher than that $\left(\sim 15,000 \mathrm{~cd} \mathrm{~m}^{-2}\right)$ of device PEI but still far lower than that $\left(121,865 \mathrm{~cd} \mathrm{~m}^{-2}\right)$ of device PEI-Zn. In addition, the maximum efficiencies of device $\mathrm{ZnO} / \mathrm{PEI}$ are inferior to those of device PEI and device PEI-Zn (see Table $\mathrm{S} 1$ and the reason is discussed in the later). As noted above, device PEI-Zn shows much better high-brightness performances compared to device PEI, device $\mathrm{ZnO} / \mathrm{PEI}$ and previously reported inverted OLEDs $[9,10,12,13,23-30]$.
Considering their EL spectra (Fig. 3d) and atomic force microscope images (Fig. S11), the high-brightness performances of device PEI-Zn are not due to spectral nor morphological variations. We attribute the high-brightness performances to the enhanced conductivity of the PEI-Zn (Fig. 2d). Firstly, as shown in Fig. S12a, the resistance of a conventional OLED (ITO/ $\mathrm{MoO}_{3}(3 \mathrm{~nm}) / \mathrm{TAPC}(30 \mathrm{~nm}) /$ TCTA $(5 \mathrm{~nm}) / \mathrm{CBP}: 10 \mathrm{wt} \% \operatorname{Ir}(\text { ppy })_{3}(30 \mathrm{~nm}) / \mathrm{TmPyPB}$ $(50 \mathrm{~nm}) / \mathrm{LiF} / \mathrm{Mg}$ : $10 \mathrm{wt} \% \mathrm{Ag}(120 \mathrm{~nm}))$ decreases from $10^{8}$ to $10^{2} \Omega$ as the bias voltage increases from 0 to $12 \mathrm{~V}$. We also measured the resistances (Fig. S12b) of the PEI and the PEI interlayers by preparing electron-only devices with a structure of ITO/PEI or PEI-Zn/DMAC-BP $(50 \mathrm{~nm}) / \mathrm{Mg}$ : $10 \mathrm{wt} \% \mathrm{Ag}(120 \mathrm{~nm})$. Figure $\mathrm{S} 12 \mathrm{~b}$ shows that even at voltage ranging from 7 to $12 \mathrm{~V}$, the PEI still has a high resistance of $10^{6} \sim 10^{8} \Omega$. Considering series circuit characteristics, device performances at high voltage would be seriously affected by the insulting property of the PEI layer (Fig. S12b). The enhanced conductivity (Figs. 2d and S12b) of the PEI-Zn is thus considered as a major reason for the performance improvements of device PEI-Zn at high voltage. It is found that the use of PEI-Zn can reduce the carrier accumulation inside the device. Figure 3e shows capacitance-voltage characteristics of the two devices. Device PEI has a $3.2 \mathrm{nF}$ capacitance increase compared to its geometric capacitance, while that for device PEI-Zn is only $1.6 \mathrm{nF}$. With the parameters of Table S1, we simulated the hole accumulations in device PEI and device PEI-Zn. It can be seen in Fig. S13 that 
the higher capacitance increase of device PEI is due to hole accumulation at the EML/TPBI and the PEI/DMAC-BPP interfaces. The accumulated holes are considered to form a built-in field across the TPBi/DMAC-BPP/PEI to enhance the electron transport of PEI layer such that device PEI still shows voltage-current density characteristics similar to device PEI-Zn at the low voltage ( $<8$ V, Fig. 3a). However, it also leads to that the PEI sustains a much higher electric field and has a lower breakdown threshold (@10 V, Fig. 3a). Interestingly, it shows that device $\mathrm{ZnO} / \mathrm{PEI}$ also breaks down at $10 \mathrm{~V}$ (Fig. S10). It supports that the PEI plays a key role in the breakdown threshold of OLEDs.

Secondly, the enhanced conductivity also allows the excitons formed at the TPBi/emitting layer (EML) interface not to be seriously quenched by triplet-polaron annihilation. We calculated the power dissipation spectra (Fig. 3f) of dipole sources at the EML/TCTA interface or the TPBi/EML interface with a homemade classic electromagnetic simulation software [32]. As shown in Fig. 3f, the peak at $u=1.22$ can be clearly attributed to surface plasmon polaritons (SPPs) at the TAPC/anode interface. Coupling of dipole radiation to the SPP mode is much weaker at the TPBi/EML interface than at the EML/TCTA interface. It leads to a much higher outcoupling efficiency (21\%) of excitons at the TPBi/EML interface compared to excitons (15\%) at the EML/TCTA interface. The CBP with a high hole mobility is thus used as the host material in this work. Besides, the reason device $\mathrm{ZnO} / \mathrm{PEI}$ has a lower efficiency due to the introduction of 30-nm ZnO which changes the waveguide modes (Fig. S10d) and leads to a lower outcoupling efficiency $(\sim 17 \%)$.

We then use a "probe detection" experiment to study the exciton distributions in the EMLs (Fig. 4a-c) and the influences of the EJLs on the exciton distributions (Fig. 4d, e). In the "probe detection" experiment, a 0.1-nm orange emitter $\operatorname{Ir}(\mathrm{MDQ})_{2}(\mathrm{acac})$ is inserted in the EML as a probe. Four devices are, respectively, prepared by placing an orange probe at $0,7,13$, and $20 \mathrm{~nm}$ from the TPBi/EML interface and marked as devices $P 1, P 2, P 3$, and $P 4$. Their EL spectra are then measured at the same current density of $3 \mathrm{~mA} \mathrm{~cm}^{-2}$, as shown in Fig. 4 b, c. Figure S14 shows that an orange probe has little influence on carrier transport characteristics of the devices. Efficient energy transfers are also anticipated from CBP and $\operatorname{Ir}(\mathrm{ppy})_{3}$ to $\operatorname{Ir}(\mathrm{MDQ})_{2}$ (acac). Thus, the ratios of orange emission to green emission can thus present the ratio of the excitons at/near the location of the orange probes to the whole excitons of the EML. The obtained exciton distributions of device PEI and device PEI$\mathrm{Zn}$ are then shown in Fig. 4d. It shows that there is a dominant exciton distribution at the TPBi/EML interface for the two devices. However, the excitons of device PEI might be seriously quenched, as the excitons ratio of device PEI is much lower than device PEI-Zn at the TPBi/EML interface. The transient EL characteristics (Fig. 4e) of device PEI and device PEI-Zn are then measured by applying a transient voltage pulse (6 V @0 48 $\mu$ s and $0 \mathrm{~V} @ 48 \sim 96 \mu \mathrm{s})$. It can be seen from Fig. 4e that device PEI-Zn shows a faster rise (left inset) and a slower decay (right inset) compared to device PEI. These results indicate excitons in device PEI$\mathrm{Zn}$ suffer from a much weaker quenching effect compared to device PEI. Nevertheless, the CBP: $\operatorname{Ir}(\mathrm{ppy})_{3}$ films on PEI or PEI-Zn/DMAC-BPP/TPBI have same PL decay characteristics (Fig. S15). It indicates that the PEI or the PEI-Zn has no directly quenching effect on the excitons of emitting layer due to the DMAC-BPP/TPBI interlayer. According to the carrier accumulations (Fig. 3e), the quenching effect is considered to be related to the triplet-polaron annihilation (TPA). As shown in Fig. S13, a higher hole accumulation is anticipated at the EML/TPBI and the DMAC-BP/PEI interfaces of device PEI to form a built-in field and enhance the electron transport of the pristine PEI. Thus, more serious TPA is anticipated in device PEI and thus a slower rise and a faster decay.

Moreover, due to the reduced carrier accumulation and the passivated amine groups $\left(-\mathrm{NH}_{2}\right.$ and $\left.-\mathrm{NH}\right)$, device PEI-Zn is also considered to show a better operating lifetime. Driven by a current density of $20 \mathrm{~mA} \mathrm{~cm}{ }^{-2}$, the operating lifetimes of device PEI and device PEI-Zn are measured in air without any protection nor encapsulation. The initial brightness $B(0)$ of device PEI and device PEI-Zn is 7,696 and 10,798 $\mathrm{cd} \mathrm{m}^{-2}$, respectively. The $\mathrm{L}_{50}$ lifetime of an OLED is defined as the time it takes until its brightness reaches $50 \%$ of the initial value. As shown in Fig. 5a, device PEI-Zn has a $\mathrm{L}_{50}$ lifetime of $5.1 \mathrm{~h}$ for $B(0)=10,798 \mathrm{~cd} \mathrm{~m}^{-2}$, which is much higher than that $\left(1.3 \mathrm{~h}\right.$ for $\left.B(0)=7696 \mathrm{~cd} / \mathrm{m}^{2}\right)$ of device PEI. The $\mathrm{LT}_{50}$ of device PEI-Zn for $B(0)=1000 \mathrm{~cd} \mathrm{~m}^{-2}$ is then extrapolated as $541 \mathrm{~h}$ by using a function $\mathrm{LT}_{50}(\mathrm{~L}) / \mathrm{LT}_{50}(\mathrm{H})=(\mathrm{H} / \mathrm{L})^{\alpha}$ with $\alpha=1.96$ [33]. It should be noted that the operating lifetime of device PEI-Zn is measured in air without any encapsulation. To our knowledge, the $\mathrm{LT}_{50}=541 \mathrm{~h}$ for $B(0)=1,000 \mathrm{~cd} \mathrm{~m}^{-2}$ of OLEDs in air (humidity, 35\%) without any encapsulation has never been reported. Besides, Fig. $5 \mathrm{~b}$ shows images of the operating device PEI and device PEI-Zn after being stored for 
(a)

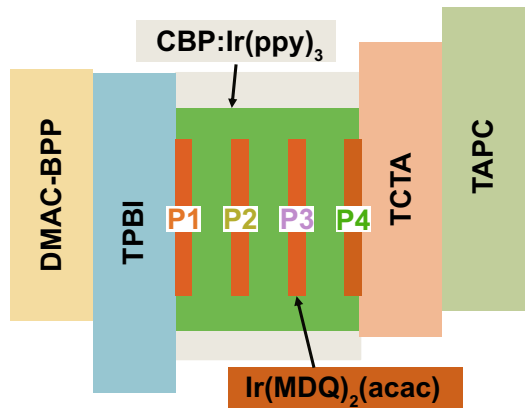

(c)

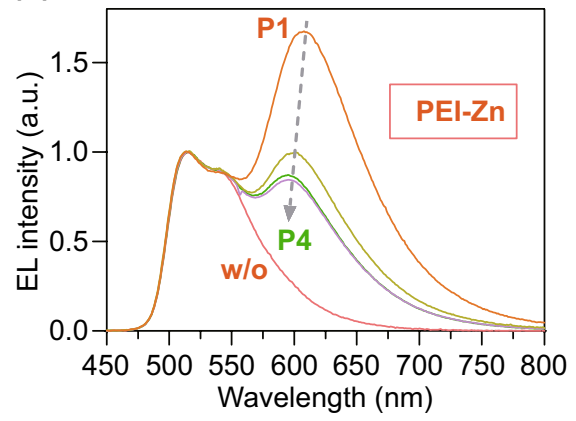

(b)

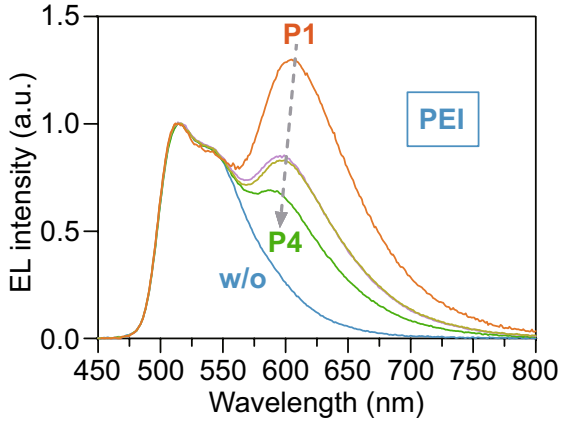

(d)

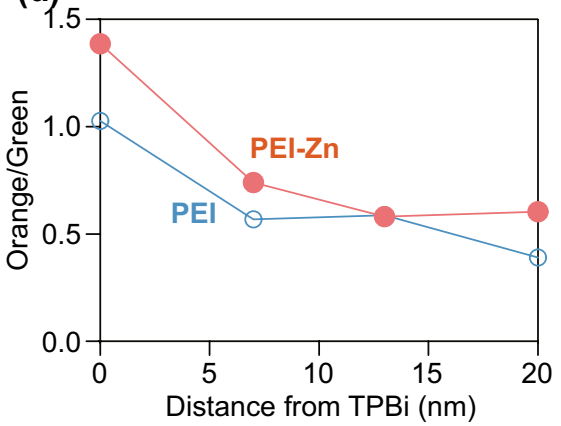

(e)

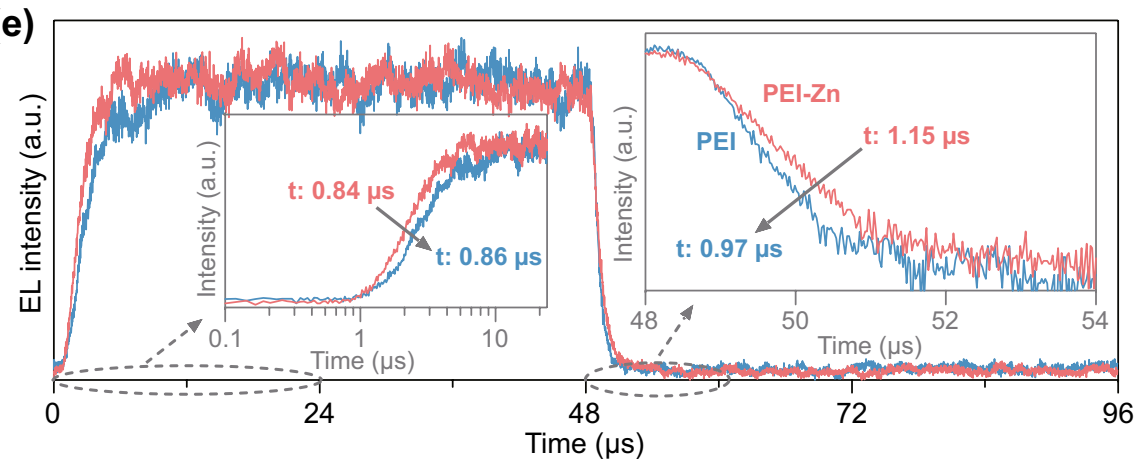

Fig. 4 a Schematic diagram of devices ( $P 1$ to $P 4)$ with an orange probe at different distance from the TPBi/EML interface. EL spectra of $\mathbf{b}$ PEIbased devices and $\mathbf{c}$ PEI-Zn-based devices with the orange probe. $\mathbf{d}$ Ratios of orange emission to green emission for PEI-based devices and PEIZn-based devices with an orange probe. e Transient EL characteristics (@520 nm) of device PEI and device PEI-Zn without the orange probe

3 and $15 \mathrm{~h}$ without any protection nor encapsulation. Due to the use of inert metal electrode, the inverted devices should exhibit much better air stability than conventional OLEDs. However, many non-emissive areas (black spots) with irregular shapes are found in device PEI. The black spots of device PEI are attributed to the loss of the ability of electron injection due to the slow reaction between PEI and organic semiconductors or ITO (Fig. 2a). On the other hand, there are only several small black spots with circle shape observed in device PEI-Zn. The regular black spot is known for researchers in the field of OLEDs as a result of the corrosion of water and oxygen to metal electrode $[34,35]$. The much fewer black spots in device PEI-Zn clearly show the advantage of the use of an inverted structure and an inert metal electrode. Our results also indicate that the use of the passivated EJL with efficient electron injection/transport is a key requirement to take the advantage of inverted OLEDs.

\subsection{Working Mechanism for PEI-Zn EJL}

As noted above, a mechanism of improvement in device PEI$\mathrm{Zn}$ is proposed in Fig. 5c, d. The improvement of device PEI-Zn is related to a self-adapting behavior of OLEDs that 

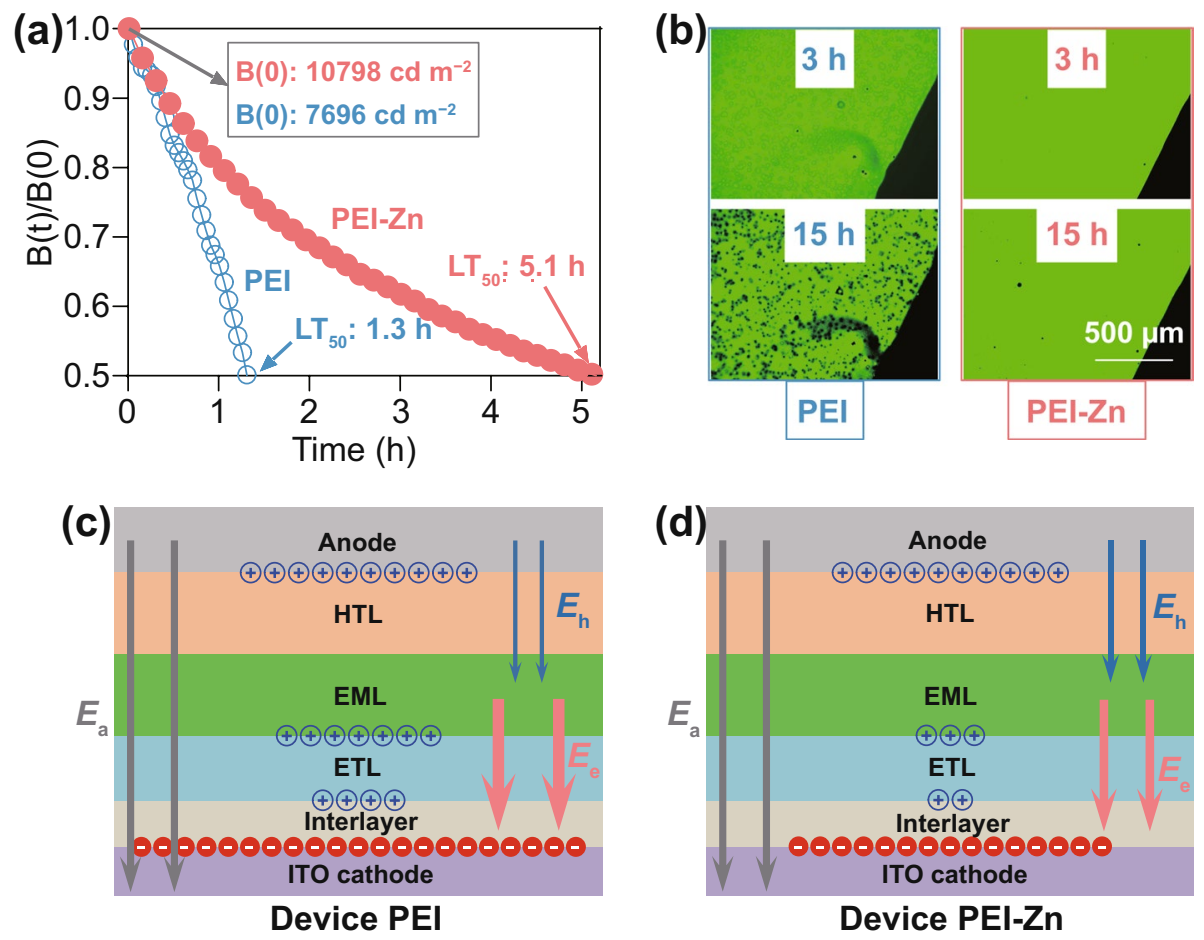

Fig. 5 a Operating lifetimes of device PEI and device PEI-Zn at a current density of $20 \mathrm{~mA} \mathrm{~cm}{ }^{-2}$. b Images of light-emitting areas of device PEI and device PEI-Zn after storing for $3 \mathrm{~h}$ and $15 \mathrm{~h}$. Schematic mechanism of $\mathbf{c}$ device PEI and $\mathbf{d}$ device PEI-Zn

enhances the balance of hole and electron currents. When the electron current is lower than the hole current, excess hole will accumulate at the interface between the EML and the electron transport layer (ETL) (Figs. 3e and S13) and affects internal electric field distribution. The increased electrical field across the ETL will then enhance the electron current to match the dominant hole current. In device PEI$\mathrm{Zn}$, the conductivity of PEI-Zn is much higher (Figs. $2 \mathrm{~d}$ and S12b) such that a reduced carrier accumulation can satisfy the need of current balance. Due to the reduced carrier accumulation, enhanced device performances are anticipated in device PEI-Zn.

\section{Conclusions}

In summary, an air-stable ultrabright inverted OLEDs is successfully prepared by using zinc ion-chelated PEI as electron injection layer. It demonstrates that the molecular orbitals of neighboring PEI chains can be fused together by the chelation between zinc ions and amine groups. It leads to two physicochemical properties of the zinc ion-chelated PEI: an enhanced conductivity and a passivated reactivity. Using the modified electron injection layer, air-stable ultrabright inverted OLEDs have been demonstrated with a recordhigh maximum brightness of $121,865 \mathrm{~cd} \mathrm{~m}^{-2}$ and an EQE over $10 \%$ at a brightness of $45,000 \mathrm{~cd} \mathrm{~m}^{-2}$. Interestingly, the inverted OLED can also show a record operating time of $541 \mathrm{~h} @ 1,000 \mathrm{~cd} \mathrm{~m}^{-2}$ in air (humidity, 35\%) for unencapsulated devices. This work paves a way for constructing high-brightness and air-stable LEDs.

Acknowledgements This work was supported by the National Natural Science Foundation of China (Grant Nos. 61905086, 62174067, 62175085), Science and Technology Development Planning of Jilin Province (Project Nos. 20190101024JH, 20200201296JC), the Hong Kong Scholars Program (Project No. XJ2020028) and grants from the Research Grants Council of the Hong Kong Special Administrative Region, China (Project Nos. 11300418 and 11300419).

Funding Open access funding provided by Shanghai Jiao Tong University.

Open Access This article is licensed under a Creative Commons Attribution 4.0 International License, which permits use, sharing, adaptation, distribution and reproduction in any medium or format, as long as you give appropriate credit to the original author(s) and the source, provide a link to the Creative Commons licence, and 
indicate if changes were made. The images or other third party material in this article are included in the article's Creative Commons licence, unless indicated otherwise in a credit line to the material. If material is not included in the article's Creative Commons licence and your intended use is not permitted by statutory regulation or exceeds the permitted use, you will need to obtain permission directly from the copyright holder. To view a copy of this licence, visit http://creativecommons.org/licenses/by/4.0/.

Supplementary Information The online version contains supplementary material available at https://doi.org/10.1007/ s40820-021-00745-w.

\section{References}

1. H.-W. Chen, J.-H. Lee, B.-Y. Lin, S. Chen, S.-T. Wu, Liquid crystal display and organic light-emitting diode display: present status and future perspectives. Light Sci. Appl. 7(3), 17168-17168 (2018). https://doi.org/10.1038/lsa.2017.168

2. T. Zhan, K. Yin, J. Xiong, Z. He, S.-T. Wu, Augmented reality and virtual reality displays: perspectives and challenges. Iscience 23(8), 101397 (2020). https://doi.org/10.1016/j.isci. 2020.101397

3. Y. Huang, E.-L. Hsiang, M.-Y. Deng, S.-T. Wu, Mini-LED, micro-LED and OLED displays: present status and future perspectives. Light Sci. Appl. 9(1), 1-16 (2020). https://doi.org/ 10.1038/s41377-020-0341-9

4. H.-M. Kim, J.G. Um, S. Lee, D.Y. Jeong, Y. Jung et al., High brightness active matrix micro-LEDs with LTPS TFT backplane. SID Symp. Dig. Tech. Pap. 49(1), 880-883 (2018). https://doi.org/10.1002/sdtp.12238

5. Y. Sun, S.R. Forrest, Enhanced light out-coupling of organic light-emitting devices using embedded low-index grids. Nat. Photonics 2(8), 483-487 (2008). https://doi.org/10.1038/nphot on.2008.132

6. S. Liu, H. Yu, Q. Zhang, F. Qin, X. Zhang et al., Efficient ITO-free organic light-emitting devices with dual-functional PSS-rich PEDOT: PSS electrode by enhancing carrier balance. J. Mater. Chem. C 7(18), 5426-5432 (2019). https://doi.org/ 10.1039/C9TC00648F

7. C.-Y. Chan, M. Tanaka, Y.-T. Lee, Y.-W. Wong, H. Nakanotani et al., Stable pure-blue hyperfluorescence organic light-emitting diodes with high-efficiency and narrow emission. Nat. Photonics 15(3), 203-207 (2021). https://doi.org/10.1038/ s41566-020-00745-z

8. J.U. Kim, I.S. Park, C.-Y. Chan, M. Tanaka, Y. Tsuchiya et al., Nanosecond-time-scale delayed fluorescence molecule for deep-blue oleds with small efficiency rolloff. Nat. Commun. 11(1), 1-8 (2020). https://doi.org/10.1038/ s41467-020-15558-5

9. S. Höfle, A. Schienle, M. Bruns, U. Lemmer, A. Colsmann, Enhanced electron injection into inverted polymer lightemitting diodes by combined solution-processed zinc oxide/ polyethylenimine interlayers. Adv. Mater. 26(17), 2750-2754 (2014). https://doi.org/10.1002/adma.201304666
10. X.-D. Zhao, Y.-Q. Li, H.-Y. Xiang, Y.-B. Zhang, J.-D. Chen et al., Efficient color-stable inverted white organic light-emitting diodes with outcoupling-enhanced $\mathrm{ZnO}$ layer. ACS Appl. Mater. Interfaces 9(3), 2767-2775 (2017). https://doi.org/10. 1021/acsami.6b14778

11. B.R. Lee, E.D. Jung, J.S. Park, Y.S. Nam, S.H. Min et al., Highly efficient inverted polymer light-emitting diodes using surface modifications of $\mathrm{ZnO}$ layer. Nat. Commun. 5(1), 1-8 (2014). https://doi.org/10.1038/ncomms5840

12. W.J. Dong, J.Y. Park, J. Ham, G.H. Jung, I. Lee et al., Dual effect of ITO-interlayer on inverted top-illuminated polymer solar cells: wetting of polyelectrolyte and tuning of cavity. Adv. Funct. Mater. 26(30), 5437-5446 (2016). https://doi.org/ 10.1002/adfm.201601764

13. H. Fukagawa, T. Sasaki, T. Tsuzuki, Y. Nakajima, T. Takei et al., Long-lived flexible displays employing efficient and stable inverted organic light-emitting diodes. Adv. Mater. 30(28), 1706768 (2018). https://doi.org/10.1002/adma.201706768

14. B.H. Lee, I.H. Jung, H.Y. Woo, H.K. Shim, G. Kim et al., Multi-charged conjugated polyelectrolytes as a versatile work function modifier for organic electronic devices. Adv. Funct. Mater. 24(8), 1100-1108 (2014). https://doi.org/10.1002/ adfm. 201301810

15. B.R. Lee, S. Lee, J.H. Park, E.D. Jung, J.C. Yu et al., Aminebased interfacial molecules for inverted polymer-based optoelectronic devices. Adv. Mater. 27(23), 3553-3559 (2015). https://doi.org/10.1002/adma.201500663

16. Y. Zhou, C. Fuentes-Hernandez, J. Shim, J. Meyer, A.J. Giordano et al., A universal method to produce low-work function electrodes for organic electronics. Science 336(6079), 327-332 (2012). https://doi.org/10.1126/science.1218829

17. F. Qin, W. Wang, L. Sun, X. Jiang, L. Hu et al., Robust metal ion-chelated polymer interfacial layer for ultraflexible nonfullerene organic solar cells. Nat. Commun. 11(1), 4508 (2020). https://doi.org/10.1038/s41467-020-18373-0

18. M. Wang, L. Zhou, M. Yu, C. Liu, S. Chu et al., Amphiphilic conjugated molecules with multifunctional properties as efficient blue emitters and cathode interlayers for inkjet printed organic light-emitting diodes. J. Mater. Chem. C 5(28), 70757083 (2017). https://doi.org/10.1039/C7TC01632H

19. Y. Gong, J. Zhang, B. Du, M. Wang, W.Y. Lai et al., Design, synthesis, and postvapor treatment of neutral fulleropyrrolidine electron-collecting interlayers for high-efficiency inverted polymer solar cells. ACS Appl. Electron. Mater. 1(6), 854-861 (2019). https://doi.org/10.1021/acsaelm.9b00016

20. W.D. Xu, W.Y. Lai, Q. Hu, X.Y. Teng, X.W. Zhang et al., A hydrophilic monodisperse conjugated starburst macromolecule with multidimensional topology as electron transport/injection layer for organic electronics. Polym. Chem. 5(8), 2942-2950 (2014). https://doi.org/10.1039/C3PY01477K

21. Z. Miao, X. Wang, R. Ma, W. Zhu, Y. Li et al., Dopamine semiquinone radical doped PEDOT: PSS: Enhanced conductivity, work function and performance in organic solar cells. Adv. Energy Mater. 10(25), 2000743 (2020). https://doi.org/ 10.1002/aenm.202000743 
22. D. Song, S. Zhao, H. Aziz, Modification of exciton lifetime by the metal cathode in phosphorescent oleds, and implications on device efficiency and efficiency roll-off behavior. Adv. Funct. Mater. 21(12), 2311-2317 (2011). https://doi.org/10. 1002/adfm.201002585

23. Y. Chen, S. Chu, R. Li, Y. Qin, Y. Xu et al., Highly efficient inverted organic light-emitting devices adopting solutionprocessed double electron-injection layers. Org. Electron. 66, 1-6 (2019). https://doi.org/10.1016/j.orgel.2018.12.008

24. W. Xu, X. Zhang, Q. Hu, L. Zhao, X. Teng et al., Fluorenebased cathode interlayer polymers for high performance solution processed organic optoelectronic devices. Org. Electron. 15(6), 1244-1253 (2014). https://doi.org/10.1016/j.orgel.2014. 03.029

25. H. Fukagawa, K. Morii, M. Hasegawa, Y. Arimoto, T. Kamada et al., Highly efficient and air-stable inverted organic light-emitting diode composed of inert materials. Appl. Phys. Express. 7(8), 082104 (2014). https://doi.org/10.7567/ APEX.7.082104

26. H. Fukagawa, M. Hasegawa, K. Morii, K. Suzuki, T. Sasaki et al., Universal strategy for efficient electron injection into organic semiconductors utilizing hydrogen bonds. Adv. Mater. 31(43), 1904201 (2019). https://doi.org/10.1002/adma.201904201

27. Y. Matsuo, H. Okada, Y. Kondo, I. Jeon, H. Wang et al., Anthracene-based organic small-molecule electron-injecting material for inverted organic light-emitting diodes. ACS Appl. Mater. Interfaces 10(14), 11810-11817 (2018). https://doi.org/ 10.1021/acsami.8b00603

28. S. Ohisa, M. Suzuki, T. Chiba, J. Kido, Doping of tetraalkylammonium salts in polyethylenimine ethoxylated for efficient electron injection layers in solution-processed organic light-emitting devices. ACS Appl. Mater. Interfaces 11(28), 25351-25357 (2019). https://doi.org/10.1021/acsami.9b06895
29. T. Chiba, Y.-J. Pu, T. Ide, S. Ohisa, H. Fukuda et al., Addition of lithium 8-quinolate into polyethylenimine electron-injection layer in oleds: Not only reducing driving voltage but also improving device lifetime. ACS Appl. Mater. Interfaces 9(21), 18113-18119 (2017). https://doi.org/10.1021/acsami.7b02658

30. J. Kim, H.-M. Kim, J. Jang, Low work function $2.81 \mathrm{eV}$ $\mathrm{Rb} 2 \mathrm{CO} 3$-doped polyethylenimine ethoxylated for inverted organic light-emitting diodes. ACS Appl. Mater. Interfaces 10(22), 18993-19001 (2018). https://doi.org/10.1021/acsami. $8 \mathrm{~b} 04760$

31. T.C. Yeh, Q. Zhu, D.B. Buchholz, A.B. Martinson, R.P.H. Chang et al., Amorphous transparent conducting oxides in context: Work function survey, trends, and facile modification. Appl. Surf. Sci. 330, 405-410 (2015). https://doi.org/10. 1016/j.apsusc.2015.01.026

32. C. Zang, S. Liu, M. Xu, R. Wang, C. Cao et al., Top-emitting thermally activated delayed fluorescence organic lightemitting devices with weak light-matter coupling. Light Sci. Appl. 10(1), 116 (2021). https://doi.org/10.1038/ s41377-021-00559-w

33. Y. Yang, Y. Zheng, W. Cao, A. Titov, J. Hyvonen et al., Highefficiency light-emitting devices based on quantum dots with tailored nanostructures. Nat. Photonics 9(4), 259-266 (2015). https://doi.org/10.1038/nphoton.2015.36

34. S. Liu, X. Zhang, S. Wang, H. Feng, J. Zhang et al., Hybrid organic light-emitting device based on ultrasonic spray-coating molybdenum trioxide transport layer with low turn-on voltage, improved efficiency \& stability. Org. Electron. 52, 264-271 (2018). https://doi.org/10.1016/j.orgel.2017.10.041

35. P. van de Weijer, K. Lu, R.R. Janssen, S.H. de Winter, H.B. Akkerman, Mechanism of the operational effect of black spot growth in OLEDs. Org. Electron. 37, 155-162 (2016). https:// doi.org/10.1016/j.orgel.2016.05.037 